\title{
Bessel function expansions of Coulomb wave functions
}

\author{
J. Humblet \\ W. K. Kellogg Radiation Laboratory, 106-038, California Institute of Technology, Pasadena, California \\ 91125 and University of Liège, Institute of Physics, B.5, Sart Tilman, B-4000 Liège 1, Belgium
}

(Received 6 March 1984; accepted for publication 12 October 1984)

From the convergence properties of the expansion of the function $\Phi_{l} \propto r^{-1-1} F_{l}$ in powers of the energy, we successively obtain the expansions of $F_{l}$ and $G_{l}$ as single series of modified Bessel functions $I_{2 l+1+n}$ and $K_{2 l+1+n}$, respectively, as well as corresponding asymptotic approximations of $G_{l}$ for $|\eta| \rightarrow \infty$. Both repulsive and attractive fields are considered for real and complex energies as well. The expansion of $F_{l}$ is not new, but its convergence is given a simpler and corrected proof. The simplest form of the asymptotic approximations obtained for $G_{l}$, in the case of a repulsive field and for low positive energies, is compared to an expansion obtained by Abramowitz.

\section{INTRODUCTION AND NOTATION}

The usual Coulomb wave functions $F_{l}$ and $G_{l}$ satisfy the differential equation

$$
\left[\frac{d^{2}}{d \rho^{2}}+1-\frac{2 \eta}{\rho}-\frac{l(l+1)}{\rho^{2}}\right] R=0
$$

in which $\rho=k r$, while $\eta$ is the Sommerfeld parameter, i.e.,

$$
\eta=\alpha / k, \quad \alpha=Z_{1} Z_{2} e^{2} M \hbar^{-2} .
$$

Let us also define the Gamov factor $C_{l}(\eta)$, the polynomial in $\eta^{-2}, u_{l}(\eta)$, and the functions $\Phi_{l}$ and $\Theta_{l}$ by the equations ${ }^{1-5}$

$$
\begin{aligned}
C_{l}(\eta)= & 2^{l} e^{-\pi \eta / 2} \\
& \times[\Gamma(l+1-i \eta) \Gamma(l+1+i \eta)]^{1 / 2} /(2 l+1) ! \\
C_{0}(\eta)= & \{2 \pi \eta /[\exp (2 \pi \eta)-1]\}^{1 / 2}, \\
u_{l}(\eta)= & \frac{\Gamma(l+1+i \eta)}{(i \eta)^{2 l+1} \Gamma(-l+i \eta)}=\frac{(2 l+1) !^{2} C_{l}^{2}}{(2 \eta)^{2 l} C_{0}^{2}} \\
= & \left(1+l^{2} \eta^{-2}\right) \cdots\left(1+2^{2} \eta^{-2}\right)\left(1+1^{2} \eta^{-2}\right), \\
u_{0}(\eta)= & 1, \\
F_{l}= & (2 l+1) ! C_{l}(\eta) \rho^{l+1} \Phi_{l}, \\
G_{l}= & (2 \eta)^{2 l+1} \rho^{l+1} \Theta_{l} /\left[(2 l+l) ! C_{l}(\eta)\right]
\end{aligned}
$$

Rewriting Eq. (1.1) in terms of the variable

$$
x=(8 \eta \rho)^{1 / 2}=(8 \alpha r)^{1 / 2},
$$

i.e., as

$$
\left[\frac{d^{2}}{d x^{2}}+\frac{d}{x d x}-1-\frac{2 l+1)^{2}}{x^{2}}+\frac{x^{2}}{16 \eta^{2}}\right]\left(\frac{R}{x}\right)=0,
$$

the latter equation is then satisfied by the functions

$$
\begin{aligned}
& (x / 2)^{2 l+1} \Phi_{l}=2 \eta C_{0}(\eta)^{-1} u_{l}(\eta)^{-1 / 2}(2 / x) F_{l}, \\
& (x / 2)^{2 l+1} \Theta_{l}=C_{0}(\eta) u_{l}(\eta)^{1 / 2}(2 / x) G_{l} .
\end{aligned}
$$

For $|\eta|=\infty, \mathrm{Eq} .(1.7)$ is satisfied by the linear combination of modified Bessel functions

$$
\alpha I_{2 l+1}(x)+b K_{2 l+1}(x),
$$

and Yost, Wheeler, and Breit ${ }^{2,3}$ have proved that

$$
\lim _{\eta=\infty} \Phi_{l}=(x / 2)^{-2 l-1} I_{2 l+1}(x),
$$

$$
\lim _{\eta=\infty} \Theta_{l}=-2(-x / 2)^{-2 l-1} K_{2 l+1}(x) .
$$

Since the modified Bessel functions $I_{n}(x)$ and $(-)^{n} K_{n}(x)$ satisfy the same differential equation and the same recurrence relations, Eqs. (1.10) suggest the existence of an expansion of $F_{l}$ and $G_{l}$ as single series of functions $I_{2 l+1+n}(x)$ and $K_{2 l+1+n}(x)$, respectively, with $n=0,1,2, \ldots$. Such expansions would be particularly useful for large $\eta$, i.e., for large $Z_{1} Z_{2}$ and/or low energies, since

$$
\eta^{-2} \propto E=\hbar^{2} k^{2} /(2 M) \text {. }
$$

Expansions of this type have been given by Abramowitz, ${ }^{6}$ the one for $G_{l}$ being asymptotic only. Since Abramowitz's derivation of these expansions is not entirely correct, we rederive the expansion of $F_{l}$ in Sec. II and simultaneously obtain the explicit form of the polynomials in $\eta^{-2}$ entering into the expansion. We obtain these results as a straightforward application of general properties we have recently established for the Kuhn-Ham expansion ${ }^{1}$ of $\Phi_{l}$. The same method is then applied in Sec. III to obtain the corresponding (exact) expansion of $G_{l}$ as a simple series in $K_{2 l+1+n}(x)$, but still as a double one in $I_{2 l+1+i}(x)$ with $0 \leqslant i \leqslant n$. For large $\eta$ and appropriate values of $\arg \eta$, the asymptotic approximation of $G_{l}$ reduces to a single series in $K_{2 l+1+n}(x)$ as it is proved in Sec. IV. This latter result is compared to that of Abramowitz, ${ }^{6}$ which also appears in the Handbook of Mathematical Functions. ${ }^{7}$ An appendix gives, up to $n=9$, the polynomials in $\eta^{-2}, b_{n}^{(l)}(\eta)$, introduced in Sec. II.

\section{EXPANSION OF THE REGULAR FUNCTION}

With the notation of Sec. I, the Kuhn-Ham expansion of $\Phi_{l}$ reads ${ }^{1}$

$$
\begin{aligned}
\Phi_{l}= & \sum_{\mu=0}^{\infty}(-)^{\mu} \eta^{-2 \mu} \sum_{\lambda=0}^{\mu} \beta_{\mu \lambda}^{(l)}\left(\frac{x}{2}\right)^{-2 l-1+2 \mu+\lambda} \\
& \times I_{2 l+1+2 \mu+\lambda}(x),
\end{aligned}
$$

where the $\beta_{\mu \lambda}^{(l)}$ are polynomials of degree $\mu-\lambda$ in $l$. They satisfy a recurrence relation also given in Ref. 1 . The expansion (2.1) is a power series of $E$, since $\eta^{-2} \propto E$. It is absolutely and uniformly convergent, both in its dependence on $\eta^{-2}$ and $x$ considered as complex variables. This property has been proved in the Appendix of Ref. 1 by obtaining a double 
series of convergent upper bounds for the modulus of each $(\mu \lambda)$ term under the conditions

$$
\left|\eta^{-2}\right|<M<\infty, \quad\left|x^{2} / 4\right|<X<\infty,
$$

the only conditions where $M$ and $X$ are arbitrarily large but bounded.

Consequently, we can rewrite the expansion of $\Phi_{l}$ as a sum over $n=2 \mu+\lambda$ and $\lambda$ without modifying its convergence properties. It then reads

$$
\Phi_{l}=\sum_{n=0}^{\infty}\left(\frac{x}{2}\right)^{-2 l-1+n} I_{2 l+1+n}(x) b_{n}^{(l)}(\eta),
$$

with

$$
b_{n}^{(l)}(\eta)=\sum_{\lambda}\left(-\eta^{2}\right)^{(n-\lambda) / 2} \beta_{(n-\lambda) / 2, \lambda}^{(l)}
$$

The latter sum extends to all integral values of $\lambda$ satisfying the conditions

$$
(n-\lambda) / 2>\lambda>0 \text {, }
$$

and having the same parity as $n$, so that $(n-\lambda) / 2$ is an integer (by definition ${ }^{1} \beta_{\mu \lambda}^{(l)}=0$ when $\mu<0$ or $\lambda<0$ or $\lambda>\mu$ ).

Accordingly, we can also write

$$
b_{n}^{(l)}(\eta)=\sum_{m=n^{\prime}}^{n^{*}}\left(-\eta^{-2}\right)^{m} \beta_{m, n-2 m}^{(l)},
$$

where ${ }^{8} n^{\prime}=[(n+2) / 3]$ and $n^{\prime \prime}=[n / 2]$. We have, in particular,

$$
b_{0}^{(l)}=1, \quad b_{1}^{(l)}=0,
$$

since $\beta_{00}^{(l)}=1$, while the inequality (2.5) cannot be satisfied for $n=1$. Introducing the expansion (2.3) into Eq. (1.7), one obtains the recurrence relation

$$
b_{n}^{(l)}(\eta)=-\frac{2 l+n}{4 \eta^{2} n} b_{n-2}^{(l)}(\eta)-\frac{1}{4 \eta^{2} n} b_{n-3}^{(l)}(\eta),
$$

for $n \geqslant 1$ and $b_{m}^{(l)}(\eta)=0$ when $m<0$.

Abramowitz's ${ }^{6}$ proof of the absolute and uniform convergence of the expansion (2.3), given explicitly for $l=0$ only, is marred by the fact that he has erroneously deduced from the relation (2.8) that $\left|b_{n}^{(0)}\right| \sim|2 \eta|^{-n}$. From Eq. (2.6), one rather has

$$
b_{n}^{(l)}(\eta)=O\left(\eta^{-2 n^{\prime}}\right), \quad \text { with } n^{\prime}=[(n+2) / 3] .
$$

From Eqs. (1.8a) and (2.3), the expansion of $F_{l}$ as a single series of modified Bessel functions reads

$$
\begin{aligned}
F_{l}= & (1 / 2 \eta) C_{0}(\eta) u_{l}(\eta)^{1 / 2} \\
& \times \sum_{n=0}^{\infty}\left(\frac{x}{2}\right)^{n+1} I_{2 l+n+1}(x) b_{n}^{(l)}(\eta) .
\end{aligned}
$$

It directly applies to a repulsive field, i.e., when $x$ is real and positive. For an attractive field, with the notation ${ }^{1}$

$$
\zeta=e^{i \pi / 2} x \quad(\xi>0), \quad \hat{\eta}=e^{i \pi} \eta=|\alpha| / k,
$$

we have $C_{0}(\eta)=C_{0}(\hat{\eta}) \exp (\pi \hat{\eta})$, and hence

$$
\begin{aligned}
F_{l}= & (-)^{l+1}(1 / 2 \hat{\eta}) C_{0}(\hat{\eta}) e^{\pi \hat{\eta}} u_{l}(\hat{\eta})^{1 / 2} \\
& \times \sum_{n=0}^{\infty}\left(\frac{-\zeta}{2}\right)^{n+1} J_{2 l+n+1}(\zeta) b_{n}^{(l)}(\hat{\eta}),
\end{aligned}
$$

i.e., a series of Bessel functions of the first kind.

\section{EXPANSION OF THE IRREGULAR FUNCTION}

We have proved in Ref. 1 that the expansion of $\Theta_{l}$, corresponding to that of $\Phi_{l}$ as given by Eq. (2.1), is

$$
\begin{aligned}
\Theta_{l}=u_{l}(\eta) & \left\{h(\eta) \Phi_{l}-\sum_{\mu=0}^{\infty}\left(-\mu^{\mu} \eta^{-2 \mu}\right.\right. \\
& \times\left[2 \sum_{\lambda=0}^{\mu} \beta_{\mu \lambda}^{(l)}\left(\frac{-x}{2}\right)^{-2 l-1+2 \mu+\lambda} K_{2 l+1+2 \mu+\lambda}(x)\right. \\
& +\sum_{s=0}^{\mu}(-)^{s} b_{s} \sum_{\lambda=0}^{\mu-s} \beta_{\mu-s, \lambda}^{(l)}\left(\frac{x}{2}\right)^{-2 l-1+2(\mu-s)+\lambda} \\
& \left.\left.\times I_{2 l+1+2(\mu-s)+\lambda}(x)\right]\right\},
\end{aligned}
$$

where $h(\eta)$ is defined in terms of psi functions $\left[\psi(z)=\Gamma^{\prime}(z) /\right.$ $\Gamma(z)]$ by

$$
h(\eta)=\frac{1}{2}[\psi(1-i \eta)+\psi(1+i \eta)]-\ln \eta,
$$

while the $b_{s}$ are defined in terms of the Bernoulli numbers ${ }^{9}$ $B_{2 s}$ by

$$
b_{0}=0, \quad b_{s}=\left|B_{2 s}\right| /(2 s) \quad(s \geqslant 1) .
$$

The function $h(\eta)$ has no convergent expansion in powers of $\eta^{-2}$, but only an asymptotic expansion for $|\eta| \rightarrow \infty$.

The series in Eq. (3.1) is absolutely and uniformly convergent under the conditions ${ }^{10}$

$$
\begin{aligned}
& \left|\eta^{-2}\right| \leqslant M<\infty, \quad|\arg x| \leqslant \pi-\epsilon<\pi, \\
& 0<\xi \leqslant\left|x^{2} / 4\right| \leqslant X<\infty .
\end{aligned}
$$

Rearranging it by introducing $n=2 \mu+\lambda$ as we have done for $\Phi_{l}$, we readily obtain the desired expansions, namely

$$
\begin{aligned}
G_{l}= & C_{0}(\eta)^{-1} u_{l}(\eta)^{1 / 2}\left\{h(\eta) \sum_{n=0}^{\infty}\left(\frac{x}{2}\right)^{n+1} I_{2 l+n+1}(x) b_{n}^{(l)}(\eta)\right. \\
& -\sum_{n=0}^{\infty}\left[2\left(\frac{-x}{2}\right)^{n+1} K_{2 l+n+1}(x) b_{n}^{(l)}(\eta)\right. \\
& +\sum_{s=0}^{[n / 2]} b_{s} \eta^{-2 s}\left(\frac{x}{2}\right)^{n+1-2 s} \\
& \left.\left.\times I_{2 l+n+1-2 s}(x) b_{n-2 s}^{(l)}(\eta)\right]\right\},
\end{aligned}
$$

for a repulsive field $(\alpha>0)$, and

$$
\begin{aligned}
G_{l}= & (-)^{l} C_{0}(\hat{\eta})^{-1} e^{-\pi \hat{\eta}} u_{l}(\hat{\eta})^{1 / 2} \\
& \times\left\{h(\hat{\eta}) \sum_{n=0}^{\infty}\left(\frac{-\zeta}{2}\right)^{n+1} J_{2 l+n+1}(\zeta) b_{n}^{(l)}(\hat{\eta})\right. \\
& +\sum_{n=0}^{\infty}\left[\pi\left(\frac{-\zeta}{2}\right)^{n+1} Y_{2 l+n+1}(\zeta) b_{n}^{(l)}(\hat{\eta})\right. \\
& -\sum_{s=0}^{[n / 2]} b_{s} \eta^{-2 s}\left(\frac{-\zeta}{2}\right)^{n+1-2 s} \\
& \left.\left.\times J_{2 l+n+1-2 s}(\zeta) b_{n-2 s}^{(l)}(\hat{\eta})\right]\right\},
\end{aligned}
$$

for an attractive field $(\alpha<0)$.

\section{ASYMPTOTIC EXPANSION OF THE IRREGULAR FUNCTION FOR LARGE $\eta$}

For real and positive $\eta$, Breit and Hull ${ }^{11}$ have proved that one obtains an asymptotic expansion of $G_{l}$ for $\eta \rightarrow \infty$ when an asymptotic expansion is substituted for $h(\eta)$. This remains valid for complex $\eta$, provided one takes into account the domain of validity of the expansion used for $h(\eta)$. From elementary properties of the $\psi$ function, ${ }^{12} h(\eta)$ is easily given the forms

$$
h(\eta)=h^{ \pm}(\eta) \mp i \pi\left(e^{2 \pi \eta}-1\right)^{-1},
$$


with $^{13}$

$$
\begin{aligned}
h^{ \pm}(\eta)= & \psi( \pm i \eta) \pm(1 / 2 i \eta)-\ln \left(e^{ \pm i \pi / 2} \eta\right) \\
& \sim \sum_{s=1}^{\infty} b_{s} \eta^{-2 s}
\end{aligned}
$$

The latter asymptotic expansion holds when $|\eta| \rightarrow \infty$ for both $h^{+}$and $h^{-}$, but, respectively, under the conditions

$|\arg \eta \pm \pi / 2|<\pi-\epsilon<\pi$.

Since $h(\eta)=\left[h^{+}(\eta)+h^{-}(\eta)\right] / 2$, we also have

$$
h(\eta) \sim \sum_{s=1}^{\infty} b_{s} \eta^{-2 s}
$$

for $|\eta| \rightarrow \infty$, but only when

$$
|\arg \eta| \leqslant \pi / 2-\epsilon<\pi / 2 \text {. }
$$

Rearranging the product $h(\eta) \Phi_{1}$ such as it is given by Eqs. (4.1), (4.3), and (2.3), we obtain, under condition (4.3b),

$$
\begin{aligned}
(x / 2)^{2 l+2} h(\eta) \Phi_{l} \sim & \mp i \pi\left(e^{2 \pi \eta}-1\right)^{-1}(x / 2)^{2 l+2} \Phi_{l} \\
& +\sum_{n=0}^{\infty} \sum_{s=0}^{[n / 2]} b_{s} \eta^{-2 s}\left(\frac{x}{2}\right)^{n+1-2 s} \\
& \times I_{2 l+n+1-2 s}(x) b_{n-2 s}^{(l)}(\eta) .
\end{aligned}
$$

Substituting this result for the first term in Eqs. (3.5), we then have, for a repulsive field $(\alpha>0)$,

$$
\begin{aligned}
G_{l} \sim & -C_{0}(\eta)^{-1} u_{l}(\eta)^{1 / 2}\left[ \pm i \pi\left(e^{2 \pi \eta}-1\right)^{-1}\right. \\
& \times \sum_{n=0}^{\infty}\left(\frac{x}{2}\right)^{n+1} I_{2 l+n+1}(x) b_{n}^{(l)}(\eta) \\
& \left.+2 \sum_{n=0}^{\infty}\left(\frac{-x}{2}\right)^{n+1} K_{2 l+n+1}(x) b_{n}^{(l)}(\eta)\right],
\end{aligned}
$$

for $|\eta| \rightarrow \infty$ and under the conditions

$$
\arg k \in[-\pi \pm \pi / 2+\epsilon, \quad+\pi \pm \pi / 2-\epsilon] .
$$

Similarly, for an attractive field $(\alpha<0)$, we have

$$
\begin{aligned}
G_{l} \sim( & -)^{l} C_{0}(\hat{\eta})^{-1} e^{-\pi \hbar \hat{\eta}} u_{l}(\hat{\eta})^{1 / 2}\left[\mp i \pi\left(e^{2 \pi \hat{\eta}}-1\right)^{-1}\right. \\
& \times \sum_{n=0}^{\infty}\left(\frac{-\zeta}{2}\right)^{n+1} J_{2 l+n+1}(\zeta) b_{n}^{(l)}(\hat{\eta}) \\
& \left.+\pi \sum_{n=0}^{\infty}\left(\frac{-\zeta}{2}\right)^{n+1} Y_{2 l+n+1}(\zeta) b_{n}^{(l)}(\hat{\eta})\right],
\end{aligned}
$$

under the same conditions $(4.6 \mathrm{~b})$.

The results just obtained take a simpler form in the right half of the complex $k$ plane, since $\left(e^{2 \pi \eta}-1\right) \sim 0$ for $|\eta| \rightarrow \infty$ in the domain (4.4b). Accordingly, we have

$$
\begin{aligned}
G_{l} \sim & -2 C_{0}(\eta)^{-1} u_{l}(\eta)^{1 / 2} \sum_{n=0}^{\infty}\left(\frac{-x}{2}\right)^{n+1} \\
& \times K_{2 l+n+1}(x) b_{n}^{(l)}(\eta),
\end{aligned}
$$

for a repulsive field and

$$
\begin{aligned}
G_{l} \sim(-)^{l} \pi C_{0}(\hat{\eta})^{-1} \mathrm{e}^{-\pi \hat{\eta}} u_{l}(\hat{\eta})^{1 / 2} \sum_{n=0}^{\infty}\left(\frac{-\zeta}{2}\right)^{n+1} \\
\quad \times Y_{2 l+n+1}(\zeta) b_{n}^{(l)}(\hat{\eta}),
\end{aligned}
$$

for an attractive one, when $k$ is real and positive, and more generally when

$$
|\arg k|<\pi / 2-\epsilon<\pi / 2 \text {. }
$$

Equations (4.7) also result directly from introducing the expansion (4.4) into Eqs. (3.5). The expansions (4.7) and the second series in the expansions (4.6) are divergent for $|\eta|^{2}<\infty$, as are the asymptotic expansions (4.3) and (4.4).

However, the expansions (4.6) and (4.7) are not asymptotic expansions proper, since any term is not always of an order of magnitude larger than the one next after it. From Eq. (2.6), it is indeed obvious that $b_{3 N-2}^{(l)}, b_{3 N-1}^{(l)}$, and $b_{3 N}^{(l)}$ are all $O\left(\eta^{-2 N}\right)$, so that the three corresponding terms in the divergent series of Eqs. (4.6) and (4.7) should actually form a single term in an asymptotic approximation of $G_{l}$. Accordingly, instead of Eq. (4.7) e.g., we must rather write

$$
\begin{aligned}
G_{l} \sim 2 & u_{l}(\eta)^{1 / 2} C_{0}(\eta)^{-1}\left\{\left(\frac{x}{2}\right) K_{2 l+1}(x)\right. \\
& -\sum_{N=0}^{\infty}\left[\sum_{i=1}^{3}\left(\frac{-x}{2}\right)^{1+3 N+i} K_{2 l+1+3 N+i}(x)\right. \\
& \left.\left.\times b_{3 N+i}^{(l)}(\eta)\right]\right\}
\end{aligned}
$$

and

$$
\begin{aligned}
G_{l} \sim & (-)^{l} \pi u_{l}(\hat{\eta})^{1 / 2} C_{0}(\hat{\eta})^{-1} e^{-\pi \hat{\eta}} \\
& \times\left\{-\left(\frac{\zeta}{2}\right) Y_{2 l+1}(\zeta)+\sum_{N=0}^{\infty}\left[\sum_{i=1}^{3}\left(\frac{-\zeta}{2}\right)^{1+3 N+i}\right.\right. \\
& \left.\left.\times Y_{2 l+1+3 N+i}(\zeta) b_{3 N+i}^{(l)}(\hat{\eta})\right]\right\},
\end{aligned}
$$

for $|\eta|=|\hat{\eta}| \rightarrow \infty$ and $|\arg k| \leqslant \pi / 2-\epsilon<\pi / 2$.

In the case of an attractive field and for real positive energies only $(\eta>0)$, the expansions (4.7a) and (4.8a) must be equivalent to the one of Breit and Hull ${ }^{11}$ and Ham, ${ }^{14}$ name$1 y,{ }^{15}$ in the notation of Secs. I and II,

$$
\begin{aligned}
G_{l} \sim & -2 u_{l}(\eta)^{1 / 2} C_{0}(\eta)^{-1} \sum_{\mu=0}^{\infty}(-)^{\mu} \eta^{-2 \mu} \\
& \times \sum_{\lambda=0}^{\mu} \beta_{\mu \lambda}^{(l)}\left(\frac{-x}{2}\right)^{2 \mu+\lambda+1} K_{2 l+1+2 \mu+\lambda}(x),
\end{aligned}
$$

for $\eta \rightarrow \infty$. Indeed, the very fact that the function

$$
G_{l} /\left[u_{l}(\eta)^{1 / 2} C_{0}(\eta)^{-1}\right]
$$

has one asymptotic power expansion in Poincarés sense entails the uniqueness of that expansion. ${ }^{16-18}$ The expansions (4.7a) and (4.9) only differ by a rearrangement of terms, the same that modified Eq. (2.1) into Eq. (2.3) and which we also used in Sec. III in absolutely and uniformly convergent double series.

In this context, it is of practical importance that we now turn to the asymptotic expansion of $G_{l}$ as given by Abramowitz, ${ }^{4,6}$ also for $\eta>0$ and $\eta \rightarrow \infty$. With the notation introduced in Sec. I, it reads

$$
\begin{aligned}
G_{l} \sim \lambda_{l}(\eta)(2 l) ! u_{l}(\eta)^{-1 / 2} C_{0}(\eta)^{-1} \\
\quad \times \sum_{n=0}^{\infty}\left(\frac{-x}{2}\right)^{n+1} K_{2 l+n+1}(x) b_{n}^{(l)}(\eta),
\end{aligned}
$$

where the overall factor $\lambda_{1}(\eta)$ remains to be fixed. Comparing Eqs. (4.7a) and (4.11), the uniqueness of the expansion of the ratio $(4.10)$ leaves no other choice than defining $\lambda_{l}(\eta)$ as being the polynomial

$$
-2 u_{l}(\eta) /(2 l) !
$$

or a function asymptotically equal to it. 
Abramowitz ${ }^{6}$ proceeds differently. He fixes $\lambda_{l}(\eta)$ by imposing the divergent expansion (4.11) to satisfy a condition ${ }^{19}$ verified by the exact (unexpanded) $G_{l}$, namely

$$
\left\{[(2 l) !]^{-1} u_{l}^{1 / 2} C_{0}(\eta)(x / 2)^{2 l} G_{l}\right\}_{x=0}=1 .
$$

This necessarily introduces in Eq. (4.11) the inverse of a divergent expansion and he actually obtains $\lambda_{l}(\eta)$ as

$$
\lambda_{l}(\eta)=-2\left[\sum_{n=0}^{\infty}(-)^{n}(2 l+n) ! b_{n}^{(l)}(\eta)\right]^{-1} .
$$

But, if we introduce the asymptotic expansion (4.7a) into Eq. (4.13), we then obtain ${ }^{20}$

$$
u_{i}(\eta) \sum_{n=0}^{\infty} \frac{(-)^{n} b_{n}^{(l)}(\eta)(2 l+n) !}{(2 l) !} \sim 1,
$$

so that the two factors (4.12) and (4.14) differ only by a factor asymptotically equal to 1 , i.e., equal to $1+O\left(\eta^{-2 N}\right)$ for any $N>1$.

The former factor (4.12) is obviously much easier to use and normally more accurate in practice, since there is no need to expand or to approximate it. It partly reduces with the factor $u_{l}^{-1 / 2}$ inEq. (4.11).

On the other hand, it is worth noticing that, for $l>0$, when only a few terms are retained in the denominator of Abramowitz's factor $\lambda_{l}(\eta)$, one introduces poles in the corresponding approximate $G_{l}$. Although, in principle, such approximations are valid only for $\eta>1$, this can, in practice, reduce the range of $\eta$ 's values in which the corresponding $G_{l}$ remains a good approximation of the exact one. ${ }^{21}$ For $l=0$, $\lambda_{l}(\eta)$ simply reduces to $\lambda_{0}=-2\left[1+O\left(\eta^{-2 N}\right)\right]$ with $N$ arbitrarily large.

To conclude, we observe that the practical advantage of the expansions (4.6)-(4.8) over those directly deduced from Ham's expansion ${ }^{3,14}$ of $G_{l}$, such as the expansion (4.9), is that they come out as single series (rather that as a double sum over $\mu$ and $\lambda)$. Moreover, the polynomials $b_{n}^{(l)}(\eta)$ can easily be obtained algebraically, by means of the single-index recurrence relation (2.8), up to any desired value of $n$. They are given in the Appendix up to $n=9$ [i.e., $N=2$ in Eq. (4.8)]. In contrast, obtaining algebraically the $\beta_{\mu \lambda}^{(l)}$ for any $l$ and $\mu$ and for $0<\lambda<\mu-1$ proved to be very tedious. ${ }^{22}$

\section{ACKNOWLEDGMENTS}

Most of this paper has been prepared at the W. K. Kellogg Radiation Laboratory of the California Institute of Technology. The author is very grateful to C. A. Barnes, W. A. Fowler, and S. E. Koonin for their warm hospitality during his stay at Caltech and their interest in this work.

\section{APPENDIX: THE FIRST POLYNOMIALS $b_{n}^{(\prime)}(\eta)$}

The polynomials $b_{n}^{(l)}(\eta)$ given hereafter for $n \leqslant 9$, i.e., $N \leqslant 2$ in Eqs. (4.8), illustrate the increase with $n$ of their low- est $\left(n^{\prime}\right)$ and highest $\left(n^{\prime \prime}\right)$ powers in $\eta^{-2}$. In agreement with Eqs. (2.4) $-(2.9)$, we have

$$
\begin{aligned}
& b_{0}^{(l)}=1, \quad b_{1}^{(l)}=0, \quad b_{2}^{(l)}=-\frac{l+1}{4 \eta^{2}}, \\
& b_{3}^{(l)}=-\frac{1}{12 \eta^{2}}, \\
& b_{4}^{(l)}=\frac{(l+1)(l+2)}{32 \eta^{4}}, \quad b_{5}^{(l)}=\frac{5 l+8}{240 \eta^{4}}, \\
& b_{6}^{(l)}=\frac{1}{288 \eta^{4}}-\frac{(l+1)(l+2)(l+3)}{384 \eta^{6}}, \\
& b_{7}^{(l)}=-\frac{35 l^{2}+147 l+142}{13440 \eta^{6}}, \\
& b_{8}^{(l)}=-\frac{5 l+11}{5760 \eta^{6}}+\frac{(l+1)(l+2)(l+3)(l+4)}{6144 \eta^{8}}, \\
& b_{9}^{(l)}=-\frac{1}{10368 \eta^{6}}+\frac{35 l^{3}+273 l^{2}+664 l+496}{161280 \eta^{8}} .
\end{aligned}
$$

Then $b_{10}^{(l)}$ and $b_{11}^{(l)}$ have terms in $\eta^{-8}$ and $\eta^{-10}$, and $b_{12}^{(l)}$ has terms in $\eta^{-8}, \eta^{-10}$, and $\eta^{-12}$. For any $n$ and $\eta \rightarrow \infty$, the main term of $b_{n}^{(l)}$ has the sign of $(-)^{n^{\prime}}$.

${ }^{1}$ J. Humblet, Ann. Phys. (N.Y.) 155, 461 (1984).

${ }^{2}$ F. L. Yost, J. A. Wheeler, and G. Breit, Phys. Rev. 49, 1 (1936).

${ }^{3}$ M. H. Hull and G. Breit, "Coulomb Wave Functions," in Encyclopedia of

Physics, edited by S. Flügge (Springer, Berlin, 1959), Vol. 41/1, Chap. 2.

${ }^{4}$ M. Abramowitz and I. A. Stegun, Handbook of Mathematical Functions

(National Bureau of Standards, Washington, D.C., 1968), 7th ed.

${ }^{5}$ The notation adopted in Eqs. $(1.5)$ is the same as in Ref. 1. It slightly differs from the one of Refs. $2-4$ for reasons given in Ref.1.

${ }^{6}$ M. Abramowitz, J. Math. Phys. 33, 111 (1954).

'See Ref. 4, Eqs. (14.4.1)-(14.4.4).

${ }^{8}[\alpha]$ is the largest integer $<\alpha$.

'See Ref. 4, Eq. (23.1.3) and Table (23.2)

${ }^{10}$ See Ref. 1, end of Sec. 5.4 and end of the Appendix.

${ }^{11}$ G. Breit and M. H. Hull, Phys. Rev. 80, 392 (1950); 80, 561 (1950).

${ }^{12}$ See Ref. 4, Eqs. (6.3.5) and (6.3.7).

${ }^{13}$ See Ref. 4, Eq. (6.3.18).

${ }^{14}$ F. S. Ham, Q. Appl. Math. 15, 31 (1957).

${ }^{15}$ Equation (4.9) is also given as Eq. (5.25) in Ref. 1, but it has been misprinted. A factor 2 is missing in its right-hand side.

${ }^{16} \mathrm{~F}$. W. J. Olver, Asymptotics and Special Functions (Academic, New York, 1974).

${ }^{17}$ In principle, one could also include the factor $u_{l}(\eta)^{1 / 2}$ in the expansion of $G_{l}$, but that requires the use of the power expansion of $u_{l}^{1 / 2}$, so that $G_{l}$ is more easily computed directly and exactly from the definition (1.4b) of $u_{i}$. The expansion of $u_{l}(\eta)$ is given in Ref. 18.

${ }^{18}$ F. S. Ham, Cruft Laboratory Technical Report 204, Harvard University, 1955, Appendix II.

${ }^{19}$ The condition (4.13) is easily obtained from Ref. 4, Eq. (14.1.14) or Ref. 1, Eq. (5.34b).

${ }^{20}$ When the exact expansion (3.5a) is introduced in Eq. (4.13), one still obtains the same relation (4.14) which contains a divergent asymptotic expansion. This is related to the fact that the second series in Eq. (3.5a) is convergent only under the conditions (3.4).

${ }^{21}$ For example, $\lambda_{1}(1)=\infty$ when terms of the order of $\eta^{-4}$ are neglected in the denominator of $\lambda_{1}(\eta)$.

${ }^{22}$ See Eqs. (3.5) in Ref. 1; $\beta_{\mu \lambda}^{(l)}$ (here) $=\left(-\mu^{\mu+\lambda} a_{\mu, 2 \mu+\lambda}^{(l)}\right.$ in Ref. 3. 